\title{
An Integrated Framework for the Development of Computer Aided Engineering Systems
}

\author{
A. Molina*, T.I.A. Ellis**, R.I.M. Young**, R. Bell** \\ *Centro de Sistemas Integrados de Manufactura, ITESM, Campus \\ Monterrey, CP 64849, Monterrey, N.L. Mexico \\ fax: +52 (8) 358-1209,e-mail: molina@mansun.lut.ac.uk \\ **Manufacturing Engineering Department, Loughborough \\ University of Technology, Loughborough, Leicestershire, LE11 3TU, \\ U.K.
}

\begin{abstract}
The development of complex engineering environments, such as Computer Aided Simultaneous Engineering Systems (CAE System), requires that system developers and users are able to hold meaningful discussions and share a common understanding of their underlying goals and ideas. A CAE Framework that aims to aid in the achievement of this goal is presented in this paper. The CAE Framework uses elements of the CIMOSA architecture framework for enterprise integration and the Reference Model for Open Distributed Processing (RM-ODP). The experience gained whilst using these reference models to define a hybrid methodology for the definition, design and implementation of CAE systems is discussed.
\end{abstract}

Keywords

CAE System, Framework, Reference Model, Simultaneous Engineering

\section{INTRODUCTION}

The development of integrated information systems to support design and manufacturing activities, and hence satisfy company requirements, represents a major problem for system developers and users. The authors have recognised that an important task in the successful 
computer support of simultaneous engineering is the identification of the enterprise requirements, i.e. where the Computer Aided Simultaneous Engineering System (CAE system) is going to be installed, what its functionality has to be and how it is operated (Molina et al. 1995). Being able to recognise these needs enables the CAE system to be configured to a specifc manufacturing environment. Different aspects which have to be considered for the elicitation of requirements are: the manufacturing strategy, the activities that require support, flow of information, availability of resources, organization and responsibilities, etc. A key problem is to determine the method, or set of methods, to be used in order to capture all these aspects in a formal representation, and hence guide the development of an integrated information system.

The IFAC/IFIP Task Force report on Architectures for Integrating Manufacturing Activities and Enterprises (Williams et al. 1993) found the following reference models suitable for the task of describing an integrated system, its life cycle and the methodology for its application: CIMOSA, GRAI-GIM and the Purdue Enterprise Reference Architecture. This Task Force aims to develop a Generic Enterprise Reference Architecture and Methodology (GERAM) on the basis of the above architectures (Bernus and Nemes 1994). Based on the concepts of reference models the authors argue that a framework or reference model is needed to design, develop and integrate CAE systems which effectively support Simultaneous Engineering. The CAE framework should assist the users and developers in the following three tasks:

1. Identify the enterprise $\mathrm{CAE}$ system requirements for the support of simultaneous engineering i.e. functionality, information, resources, role in the organization, etc.

2. Guide the design and implementation of the CAE system itself.

3. Organize the people and establish the set of methods and tools to evolve the CAE system towards the desired level of integration and automation.

This paper introduces a CAE framework which aims to address these issues by combining the use of two reference models: the Open System Architecture for Computer Integrated Manufacturing-CIMOSA (ESPRIT Project 688/5288) and the Reference Model for Open Distributed Processing, named RM-ODP (ISO/IEC JTC1/SC21/WG7 N 755).

The paper is organized as follows, the next section describes in detail the issues involved in the definition of the CAE framework. The use of the CIMOSA model is presented in Section 3. Section 4 describes the utilization of the RM-ODP to define what has been called the MOSES CAE Reference Model. A discussion about the importance of using reference models for the definition, design and implementation of CAE systems is offered in section 5. Finally conclusions are proffered on the authors' experiences in the use of reference models.

\section{A CAE FRAMEWORK FOR COMPUTER AIDED SIMULTANEOUS ENGINEERING SYSTEMS}

The rationale of the CAE Framework is based on the idea that a formal definition of enterprise requirements can be used as the driver for the selection of alternative CAE systems. The match between the particular needs of an enterprise against different available CAE systems can be 

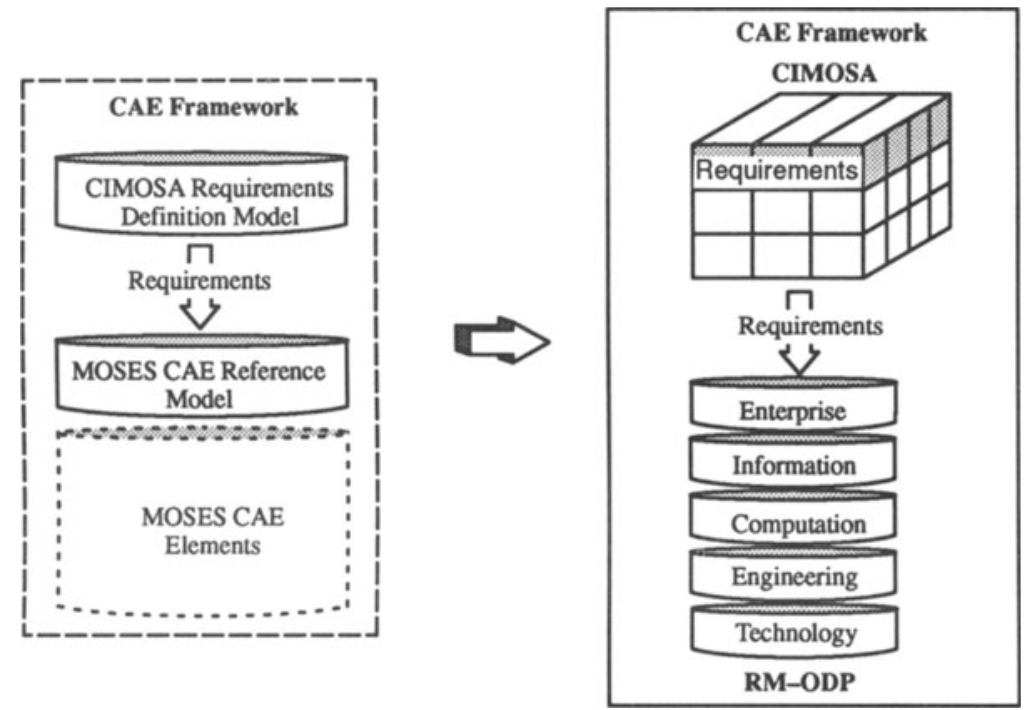

Figure 1 CAE Framework based on CIMOSA Requirements Definition Model and Reference Model for Open Distributed Processing (RM-ODP).

facilitated, if the CAE system concepts are represented in a reference model which allows the description of the CAE systems functionality and elements. This idea of using a model to define the enterprise requirements has been introduced by the authors in the MOSES research project in order to define the CAE framework. The use of a requirements model allows different enterprises to specify what functionality is required of a CAE system in order to support simultaneous engineering. To define the enterprise requirements in a formal manner, the authors decided to use the Requirements Definition Model of the CIMOSA architectural framework. The enterprise requirements are those needed to realize the design of products using simultaneous engineering principles. The main reason for this choice was that CIMOSA provides a set of methods to systematically develop the requirements model.

The CAE Framework is therefore composed of two reference models (Figure 1):

1. The CIMOSA Requirements Definition Model

2. The MOSES CAE Reference Model based on the Reference Model for Open Distributed Processing (RM-ODP)

The Reference Model for Open Distributed Processing (RM-ODP) was used to define the MOSES CAE Reference Model (CAE-RM) in order to provide a multi-viewpoint representation of the MOSES CAE system concepts (Molina et al. 1994). The CAE-RM describes the functionality, configuration and technology necessary to satisfy the requirements specified by the CIMOSA Requirements Definition Model. 


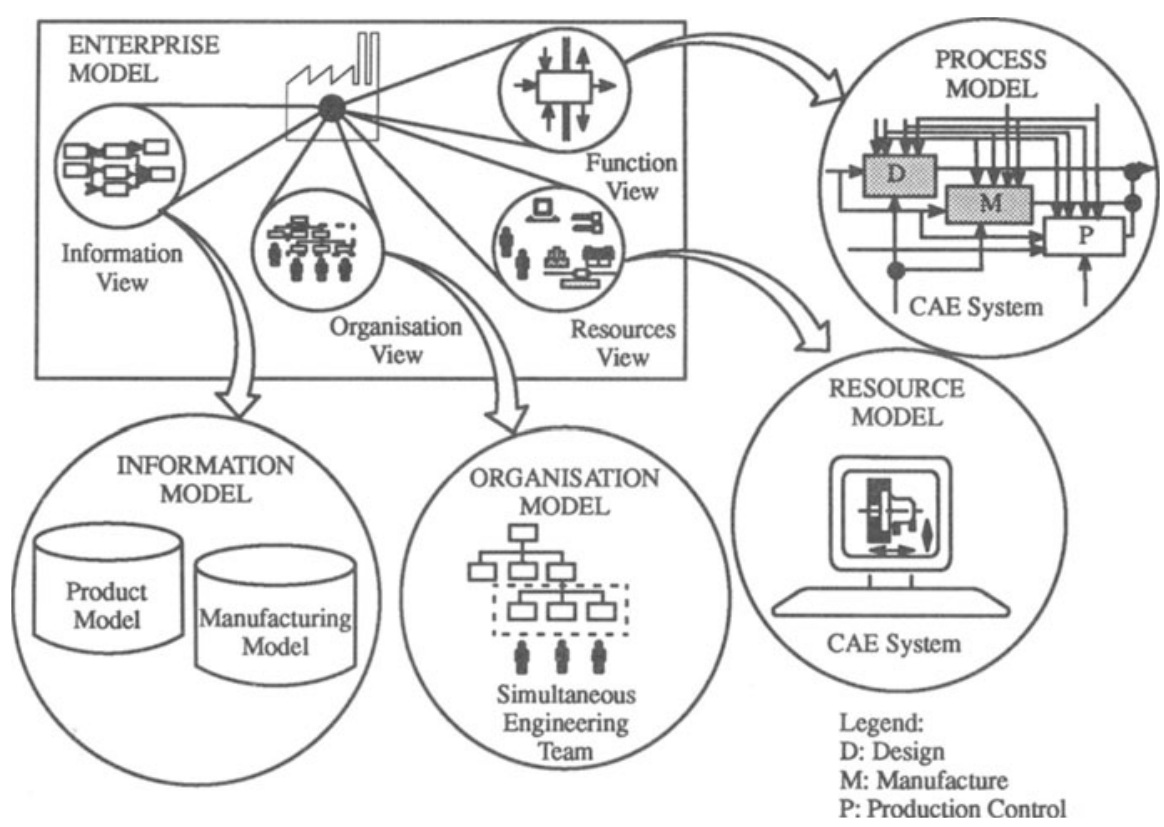

Figure 2 CIMOSA Partial Enterprise Requirements Definition Model.

The CAE Framework is therefore an hybrid methodology based on the CIMOSA Requirements Definitions Model and the multi-viewpoint MOSES CAE-RM based on RM-ODP. This CAE Framework aims to enable the identification of requirements for a CAE system, the provision of guide-lines for CAE system development and the organisation of methods and tools to evolve the CAE system towards the required level of integration and automation.

\section{THE USE OF CIMOSA IN THE CAE FRAMEWORK}

The CIMOSA Reference Model allows the construction of enterprise models. In order to build the CIMOSA Requirements Definition Model model the authors followed the CIMOSA modelling process which requires the enterprise model to be built in terms of four interrelated views: function, information, resources and organisation (Figure 2). By modelling the function view, the activities to design, produce and maintain products with consideration for the whole product life-cycle are identified. The model developed in this research only covers the activities relevant to the development of products using simultaneous engineering principles, and not all the activities in the enterprise. During the modelling of these activities the information required to realize these activities is determined. This information is modelled and captured in the information view by using information models. By combining the results of the information and function views the required capabilities for the CAE system to support simultaneous engineering, as a resource, can be defined. Thus, the CAE system capabilities form the resource view. The 
organisation view models the responsibilities and authorities of the group of people who have influence over the elements defined in the function view, information view and resource view (in this case, the Simultaneous Engineering Team). This team is responsible for the realization of the concurrent design of products.

Once the Partial CIMOSA Requirements Definition Model was defined, this model was instantiated to specify the CAE system requirements for a particular enterprise. The CIMOSA model is therefore a driver for the definition of the functions required by a CAE system. The use of CIMOSA allows the creation of a more structured and flexible CAE Framework. These characteristics enable the framework to be based upon an available, acceptable and formal terminology and methodology; and perhaps a future standard. In addition, the model can be applied to a wide range of systems within the domain of information systems to support simultaneous engineering. The use of CIMOSA in the CAE Framework allows future CAE systems, developed based on the MOSES CAE-RM, to be easily integrated within the enterprise. This integration can be more easily achieved because the system requirements are clearly defined and may be used as the drivers for the development of the CAE system.

The author's main reasons for chosing the CIMOSA reference model as part of the CAE Framework were: it is a well defined and documented reference model, it has a formal approach to system modelling, the CIMOSA concepts match the ones required for the definition of the $\mathrm{CAE}-\mathrm{RM}$ at the enterprise view, it has the potential to become an international standard

\section{THE USE OF RM-ODP IN THE CAE FRAMEWORK}

Once all the enterprise requirements for the computer support of simultaneous engineering have been identified and defined using CIMOSA, the context for the development of the CAE system, based on the MOSES CAE Reference Model (CAE-RM), is set. The aim of the CAE-RM is to provide a description of a CAE system and its functionality that matches and satisfies those requirements, and then to guide the development of the CAE system itself. On this basis, the design, configuration and implementation of the CAE system can be undertaken by selecting the system elements, defined in the CAE-RM, which are important for that particular manufacturing environment such as: information models (e.g. Product and Manufacturing Models), decision support environments (e.g. applications for Design for Function, Design for Manufacture, etc.), and the adequate integration infrastructure (i.e. information system architecture).

The CAE-RM is based on the RM-ODP (ISO/IEC JTC1/SC21/WG7 N 755), which is a five level model intended to represent open distributed systems. To achieve this, the following five levels have been defined: Enterprise, Information, Computation, Engineering, and Technology. Although the RM-ODP allows the thorough description of a CAE system from different views, the MOSES research focused primarily in defining the first three viewpoints i.e. Enterprise, Information and Computation (Figure 3).

The purpose of using the CAE-RM is to model a real system, whether existing or planned. The enterprise may have requirements which are difficult to realise. Any gap between what can be achieved by the system and what is desired will be highlighted by a disparity in the mapping between the CIMOSA Enterprise Requirements Definition Model and the Enterprise viewpoint of the CAE-RM. 


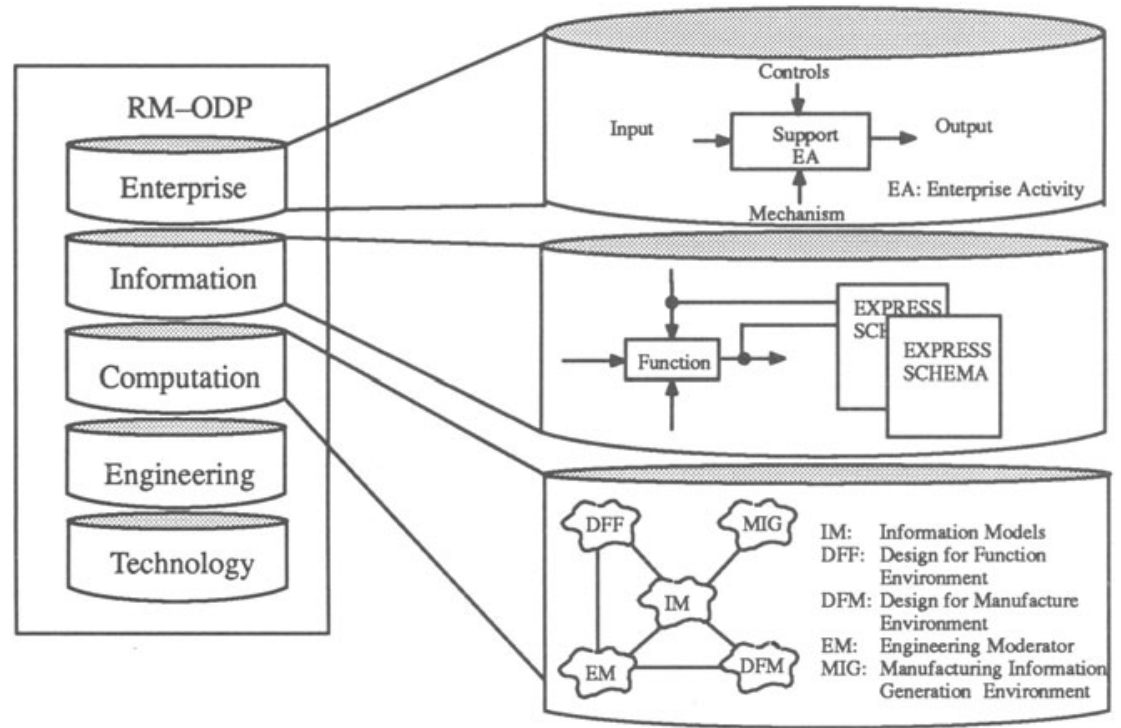

Figure 3 The MOSES CAE Reference Model.

In the MOSES CAE Reference Model the Enterprise Viewpoint is described using IDEF0 activity diagrams (ICAM 1980). The IDEF0 model describes the purpose of the CAE system, i.e. support the enterprise activities undertaken by the simultaneous engineering team. The Enterprise Viewpoint of the CAE-RM allows the establishment of a relationship between the CIMOSA Requirements Definition Model and the CAE-RM. This relationship is created between an enterprise activity defined in the CIMOSA model and an activity defined in the IDEF0 model of the Enterprise Viewpoint. Each enterprise activity is defined in terms of inputs and outputs. The CIMOSA resource inputs define the capabilities that are required from a resource in order to perform certain activities. Thus the specific CAE system capabilities which are needed to realize certain enterprise activities are defined and from this definition the CAE system requirements are derived. The IDEF0 activity diagrams of the Enterprise Viewpoint describe the functions of the system which can satisfy the enterprise requirements, and therefore the CIMOSA enterprise activities which can be supported.

The information flows, together with information structures, are represented at the Information Viewpoint. In the CAE-RM this viewpoint is defined via a combination of IDEF0 models and EXPRESS (ISO 10303-11). This combination of models allows the description of the information flows together with the structure of the information elements, their relationships and relevant attributes.

The description of the Computational Viewpoint is based on the Booch Methodology (Booch 1991). This methodology seems to fulfil the requirements needed to describe this view, i.e. define all the computational objects within the CAE system, the activities that occur within those objects 
and the interactions among the objects. The MOSES CAE system comprises the following objects: Information Models, Design for Function Environment, Design for Manufacture Environment, Engineering Moderator, and Manufacturing Information Generation Environment. These objects are required to design and implement MOSES type CAE systems as object oriented information systems with the characteristics of being modular, flexible and open.

The Engineering Viewpoint focuses on the infrastructure required to support distribution. This view enables the specification of the processing, storage and communication functions required to implement the system. This viewpoint, in our case, is supported by defining an Integration Environment which supports remote procedure call functions. The CIMOSA integrated infrastructure can be used as the basis for the definition of this viewpoint in order to enable the integration of the MOSES CAE System with other CIMOSA based systems. Finally, the Technological Viewpoint focuses on the selection of the necessary technology to support the system. In this research the object oriented database DEC Object/DB and the object oriented programming language $\mathrm{C}++$ are being used.

\section{DISCUSSION ON THE USE OF REFERENCE MODELS}

Increasing the levels of automation in information systems for the support of design and manufacturing activities is a key issue that must be tackled jointly by users and developers. These information systems should provide decision support and offer significant advantages for the improvement of product development activities. The systems are intended to assist human decision makers exercise judgement. A consequence of this is that the complexity of data, information and knowledge that must be modelled and used by a CAE system increases dramatically. The management of this complexity is an onerous task, and tools and methodologies to help support system development are essential if the current pace of system evolution is to be maintained or improved.

The authors' experience in developing a computer system to support simultaneous engineering activities has allowed them to recognise two key issues in integrated system development: the importance of identifying the real enterprise requirements for information system support, and the need to design and implement systems which are easily configured, standardized and integrated. These issues have been addressed in the MOSES research project by using the CAE framework in order to achieve the required system configuration and by considering the particular requirements of each company involved in the MOSES project. Different configurations of the MOSES CAE system have been developed to tackle the specific problems faced by each of the industrial collaborators.

The CAE Framework allows assessment of the ways in which a particular CAE system, developed based on the CAE-RM, could provide support to different CIMOSA enterprise requirements models which each represent the needs of a particular firm. The Partial CIMOSA Enterprise Requirements Definition Model, developed in this research, can be instantiated to represent the requirements of a company and then the functionality of the MOSES CAE-RM is matched to this model in order to establish the important link between requirements to be satisfied, and the functions provided in the system to satisfy them. This approach has proven to be successful in the case studies carried out to date. 


\section{CONCLUSIONS}

The combined use of CIMOSA and RM-ODP in the CAE Framework has enabled the support of two tasks: identification of requirements for CAE systems in an enterprise, and the introduction of guide-lines for CAE system design and development. However, the organization and management of the timely incorporation of people, methods and tools to evolve the CAE system environment towards the concept of an integrated enterprise requires further research.

\section{ACKNOWLEDGEMENTS}

The research is part of an ACME funded project, 'Exploiting Product and Manufacturing Models in Simultaneous Engineering', pursued at Loughborough University of Technology and Leeds University, and supported by a group of industrial collaborators [SERC ref. GR/H 24273 and GR/H 24266 respectively]. The research undertaken by A. Molina is funded by the Mexican Government (CONACyT), Monterrey Institute of Technology (ITESM, Mexico) and ORS Award Scheme (ORS/9226010).

\section{REFERENCES}

Bernus, P. and Nemes, L. (1994) A Framework to Define a Generic Enterprise Reference Architecture and Methodology. Draft proposal, IFAC/IFIP Task Force on Architectures for Enterprise Integration, Eight Workshop Meeting, Vienna, Austria, June 11-12.

Booch, G. (1991) Object-oriented design with applications, Benjamin/Cummings Inc.

ESPRIT Project 688/5288, AMICE, Volume 1, CIMOSA: Open System Architecture for CIM, ESPRIT Consortium AMICE (Eds.), 2nd, revised and extended edition, Springer-Verlag, 1993.

ICAM (1980) Architects' Manual ICAM Definition Method (IDEF0), DR-80-ATPC ol.

ISO CD 10303-11, Industrial automation systems and integration-Product data representation and exchange - Part 11: Description methods: The EXPRESS language reference manual.

ISO/IEC JTC1/SC21/WG7 N 755 (1993) 'Draft Recommendation X.901: Basic Reference Model of Open Distributed Processing - Part 1: Overview and guide to use", ISO/IEC JTC1/SC21/WG7.

Molina, A., Ellis, T.IA., Young, R.I.M. and Bell, R. (1994) Methods and Tools for Modelling Manufacturing Information to Support Simultaneous Engineering, Preprints, 2nd IFAC/IFIP/IFORS Workshop Intelligent Manufacturing Systems - IMS'94, (Ed. P. Kopacek), Vienna - Austria - June 13-15, 1994, pp. 87-93.

Molina, A. et al. (1995) A Review of Computer Aided Simultaneous Engineering Systems, Research in Engineering Design 7:38-63.

Williams, T.J. et al. (1993) Architectures for Integrating Manufacturing Activities and Enterprises, in H. Yoshikawa and J. Goossenaerts (eds.), Information Infrastructure Systems for Manufacturing, Proceedings, JSPE/IFIP TC5/WG5.3 Workshop on the Design of Information Infrastructure Systems for Manufacturing, DIISM'93, Tokyo, 8-10 November, 1993, North-Holland, pp. 3-16. 


\section{BIOGRAPHY}

Dr Arturo Molina is an Associate Professor in the Integrated Manufacturing System Center at Monterrey Institute of Technology (ITESM), Campus Monterrey, Mexico. He received his University Doctor degree in Mechanical Engineering at the Technical University of Budapest in November 1992, and his M.Sc. degree in Computer Science from ITESM, Campus Monterrey in December 1990. He is a $\mathrm{PhD}$ Candidate in Manufacturing Engineering at Loughborough University of Technology. His current research interests include information systems support for concurrent engineering, information modelling, enterprise integration, enterprise modelling, and design and planning of manufacturing systems.

Mr. Tim Ellis is a research associate on the MOSES project. He is primarily involved in determining how best to support design for manufacture in a data model environment. This encompases product and manufacturing modelling and expert application development. He holds a degree in Computer Aided Engineering and an MSc in Computer Integrated Manufacture. His work experience includes time in the structural mechanics and computational physics departments of a large engineering consultancy and the development of multimedia application prototypes for a large automotive manufacturer.

A Mechanical Engineer by training, Dr. R.I.M. Young is currently a lecturer in the Department of Manufacturing Engineering at Loughborough University of Technology. His research interests are in integrated design and manufacture, and software support for concurrent engineering. Prior to joining Loughborough in 1985 he worked for some 10 years in industry, being mainly concerned with the design and development of new products and processes. Dr. Young's recent research publications have addressed issues in product modelling, features technology, process planning, design for manufacture and modelling manufacturing information.

Professor Robert Bell graduated from UMIST in Electrical Engineering in 1954, and with a Masters degree in Textile Technology in 1956. Graduate trainee at Metropolitan Vickers 1956-58; research engineer at UMIST in machine tool research 1958-60. Teaching and research in the field of machine tool engineering at UMIST 1960-75. Appointed Reader in 1972; awarded DSc in 1975. Appointed Professor of Manufacturing Technology at LUT in 1978. Established research in flexible manufacturing systems with particular emphasis given to modelling methods for cell design. Contemporary research interests concerned with the role of product and manufacturing models in computer integrated engineering, concepts for factory modelling, and research into tool management systems. A major additional interest over the last three years has been the international activities in IMS, being a member of the European and International Technical Committees throughout the feasibility study. A further major interest has been support for academic development under the aegis of the ODA and UN, with assignments in Brazil, Hong Kong, India, Mexico, Singapore, Sri Lanka and Turkey. 